\title{
miR-146b correlates with increased disease activity and psoriatic tissue inflammation and promotes keratinocyte proliferation in psoriasis
}

\author{
LING ZHANG $^{1}$, SHENGLAN ZHANG $^{2}$, JIANBIN WANG ${ }^{1}$ and XIAOJING LI ${ }^{1}$ \\ ${ }^{1}$ Department of Dermatology, Affiliated Hospital of Hebei University of Engineering; \\ ${ }^{2}$ Medical Department, Handan Central Hospital, Handan, Hebei 056002, P.R. China
}

Received May 24, 2019; Accepted October 1, 2020

DOI: $10.3892 /$ etm.2021.9727

\begin{abstract}
The present study aimed to investigate the expression of microRNA (miR)-146b in psoriatic tissue and its correlation with psoriasis activity and inflammation. The effect of miR-146b overexpression on keratinocyte proliferation and apoptosis was also explored. The expression of miR-146b in the psoriasis-affected tissue and non-affected tissue of 110 patients was determined via reverse transcription-quantitative (RT-q)PCR. The psoriasis-affected body surface area and psoriasis area severity index (PASI) score were recorded for evaluating disease activity. The expression of various inflammatory cytokines in psoriasis-affected tissue was also detected via RT-qPCR. miR-146b overexpression and control plasmids were constructed and transfected into $\mathrm{HaCaT}$ cells in vitro. Subsequently, cell proliferation, apoptosis and tumor necrosis factor (TNF)-related apoptosis-inducing ligand (TRAIL)-induced cell apoptosis were determined. The results revealed that the expression of miR-146b was increased in psoriasis-affected tissue compared with that in unaffected tissue. The results obtained from a receiver operating characteristic curve analysis demonstrated that miR-146b levels were able to discriminate between psoriasis-affected tissue and unaffected tissue, with an area under the curve value of 0.781
\end{abstract}

Correspondence to: Professor Xiaojing Li, Department of Dermatology, Affiliated Hospital of Hebei University of Engineering, 81 Congtai Road, Handan, Hebei 056002, P.R. China E-mail: huan91439141@163.com

Abbreviations: miRNAs, microRNAs; RA, rheumatoid arthritis; ATD, autoimmune thyroid diseases; BMI, body mass index; BSA, body surface area; PASI, psoriasis area and severity index; TNF- $\alpha$, tumor necrosis factor $\alpha$; IL, interleukin; TRAIL, TNF-related apoptosis-inducing ligand; ROC, receiver operating characteristic; AUC, area under curve; TRAF6, TNF receptor-associated factor 6; IRAK-1, IL-1 receptor-associated kinase 1; imDCs, immature dendritic cells; mDCs, mature DCs

Key words: psoriasis, microRNA-146b, disease activity, inflammation, keratinocyte
(95\% CI: 0.720-0.843). In addition, miR-146b expression in psoriatic tissue was correlated with an increased PASI score in patients with psoriasis. miR-146b expression in psoriatic tissue was positively correlated with TNF- $\alpha$, interleukin (IL)-6 and IL-17 mRNA levels. In vitro, miR-146b overexpression enhanced $\mathrm{HaCaT}$ cell proliferation and suppressed apoptosis as well as TRAIL-induced apoptosis when compared with that in control-transfected HaCaT cells. In conclusion, miR-146b was positively correlated with disease activity and psoriatic tissue inflammation. Keratinocyte proliferation was also promoted in psoriasis.

\section{Introduction}

Psoriasis is a common autoimmune disease that is characterized by thick erythematous skin plaques, affecting $0.91-8.5 \%$ of the global population $(1,2)$. The high prevalence and long disease course of psoriasis cause substantial economic and psychological burdens to individuals and their families $(3,4)$. In addition, psoriasis is a type of chronic inflammatory condition and is classified as a T-helper (Th)1 disease, as the cytokines involved in the Th1 pathway [including tumor necrosis factor $\alpha$ (TNF- $\alpha$ ), interleukin (IL)-6, IL-17 and IL-22 expression] are increased in the lesional skin and peripheral blood of patients (5). Although several therapeutic approaches (including topical therapy, phototherapy, systemic non-biologic treatments and biologic treatment) are able to decrease the disease activity in the majority of circumstances, there is still no cure for psoriasis $(6,7)$. Therefore, it is paramount to investigate the underlying mechanisms of the pathology of psoriasis to further explore potential treatment options.

MicroRNAs (miRNAs/miRs) are a group of small non-coding RNAs comprised of 22 nucleotides (8). Accumulating studies have revealed that miRNAs are widely distributed in animals, plants and certain viruses, and they have been determined to be key regulators of numerous important biological processes, including cell proliferation, differentiation and inflammation (9-11). As one of the commonly investigated miRNAs, miR-146b has been implicated in the malignant proliferation of various cancer cell types (including ovarian and papillary thyroid cancer cells) and several immune activities, including innate immune 
sensing and pro-inflammatory cytokine release (12-16). Several studies have revealed that miR-146b expression is increased in several autoimmune diseases [including rheumatoid arthritis (RA), multiple sclerosis and autoimmune thyroid diseases (ATD)] (17-19). Considering that miR-146b promotes cell proliferation and has a role in the pathology of several autoimmune diseases, and that psoriasis is a typical autoimmune disease associated with malignant proliferation of keratinocytes, the present study hypothesized that miR-146b may participate in the development and progression of psoriasis. Of note, the role of miR-146b in the pathology of psoriasis has so far remained elusive. Therefore, the present study aimed to explore the expression of miR-146b in psoriatic tissue and to determine its association with psoriasis activity and inflammation. In addition, the present study explored the effect of miR-146b overexpression on the regulation of keratinocyte proliferation and apoptosis.

\section{Materials and methods}

Patients. A total of 110 patients with psoriasis were enrolled at the Affiliated Hospital of Hebei University of Engineering (Handan, China) between January 2017 and June 2018. The inclusion criteria were as follows: i) Diagnosed with psoriasis vulgaris; ii) age $>18$ years; iii) consent to donate psoriasis-affected skin tissue samples and normal skin tissue samples. The exclusion criteria were as follows: i) Complicated with inflammatory skin diseases other than psoriasis; ii) complicated with other systemic immune or inflammatory diseases, including systemic lupus erythematosus or inflammatory bowel disease; iii) complicated with malignancies. The present study was approved by the Ethics Review Board of the Affiliated Hospital of Hebei University of Engineering (Handan, China). All patients provided written informed consent.

Disease assessment. After enrollment, the characteristics of the patients, including their age, gender, body mass index (BMI), disease duration and treatment options, were documented. The psoriasis-affected body surface area (BSA) and psoriasis area severity index (PASI) scores were subsequently determined $(20,21)$.

Sample collection and reverse transcription-quantitative $(R T-q) P C R$. Psoriasis-affected skin and normal skin tissue samples were obtained via punch biopsy. The expression of miR-146b, TNF- $\alpha$, IL-6, IL-17 and IL-22 in each sample were measured via RT-qPCR. Total RNA was extracted using TRIzol reagent (Invitrogen; Thermo Fisher Scientific, Inc.), after which the concentration, purity and integrity of samples was determined. Complementary DNA was then produced by RT using the ReverTra Ace ${ }^{\circledR}$ qPCR RT kit (Toyobo Life Science). PCR was performed using SYBR ${ }^{\circledR}$ Green Realtime PCR Master mix (Toyobo Life Science). The primers utilized are listed in Table I. The quantitative results of the PCR analysis were calculated using $2^{-\Delta \Delta C q}$ method (22). U6 was used as a reference gene for miR-146b, while GAPDH was used as a reference for the remaining mRNAs. The RT was performed using the following conditions: $65^{\circ} \mathrm{C}$ for $5 \mathrm{~min}, 37^{\circ} \mathrm{C}$ for $20 \mathrm{~min}$ and then $98^{\circ} \mathrm{C}$ for $5 \mathrm{~min}$. The qPCR amplification was performed using the following program: $95^{\circ} \mathrm{C}$ for $60 \mathrm{sec}$, followed by 40 cycles of $95^{\circ} \mathrm{C}$ for $15 \mathrm{sec}$ and $61^{\circ} \mathrm{C}$ for $30 \mathrm{sec}$.

Cell culture and transfection. The spontaneously immortalized human keratinocyte cell line $\mathrm{HaCaT}$ was purchased from Kunming Cell Bank and cultured in Dulbecco's modified Eagle's medium (Gibco; Thermo Fisher Scientific, Inc.) supplemented with $10 \%$ inactivated fetal bovine serum (Gibco; Thermo Fisher Scientific, Inc.), penicillin (100 U/ml) and streptomycin $(100 \mathrm{mg} / \mathrm{ml}$; both from Sigma-Aldrich; Merck KGaA). miR-146b overexpression and control overexpression plasmids (with non-coding DNA fragment) were constructed by Shanghai QeeJen Bio-Tech Co., Ltd. and then transfected into $\mathrm{HaCaT}$ cells, which were correspondingly designated as the miR-146b+ group and the Control+ group. The relative expression of miR-146b was detected at $24 \mathrm{~h}$ post-transfection via RT-qPCR as described above. All cell experiments were performed in triplicate.

Measurement of cell proliferation and apoptosis. After transfection, cell proliferation was detected at $0,24,48$ and $72 \mathrm{~h}$ using a Cell Counting Kit-8 (Dojindo Molecular Technologies, Inc.). The rate of cell apoptosis was also detected at $24 \mathrm{~h}$ following transfection with an FITC Annexin V Apoptosis Detection kit II (BD Biosciences). The flow cytometer (BD Biosciences) was used for the Annexin V assay. All procedures were performed according to the manufacturers' protocols.

Measurement of TNF-related apoptosis-inducing ligand (TRAIL)-induced apoptosis. At $48 \mathrm{~h}$ post-transfection, $200 \mathrm{ng} / \mathrm{ml}$ TRAIL (Sigma-Aldrich; Merck KGaA) was added to $\mathrm{HaCaT}$ cells and the cells were incubated for a further $24 \mathrm{~h}$. The rate of cell apoptosis was then detected using an FITC Annexin V Apoptosis Detection kit II (BD Biosciences) to determine TRAIL-induced apoptosis.

Statistical analysis. SPSS Software 23.0 (IBM Corp.) was used for statistical analysis and GraphPad Prism Software 7.01 (GraphPad, Inc.) was used for visualization of quantitative results. Comparisons between two groups were performed using Wilcoxon's signed rank-sum tests. Comparisons between two individual groups were determined by Wilcoxon's rank-sum tests and Student's t-tests. Differences in miR-146b expression among groups were determined using the Kruskal-Wallis H-test. Correlations between two parameters were determined by Spearman's correlation test. In addition, receiver operating characteristic (ROC) curves were drawn and the area under the curve (AUC) was calculated to determine the ability of the parameter to distinguish psoriasis-affected skin tissue from normal skin tissue. $\mathrm{P}<0.05$ was considered to indicate a statistically significant difference.

\section{Results}

Characteristics of patients. The characteristics of the patients are presented in Table II. The mean age of the 110 patients with psoriasis included in the present study was $47.4 \pm 10.8$ years, and the cohort comprised $65(59.1 \%)$ males and $45(40.9 \%)$ females. The mean psoriasis-affected BSA 
Table I. Primer list.

\begin{tabular}{lll}
\hline Gene & \multicolumn{1}{c}{ Forward primer } & \multicolumn{1}{c}{ Reverse primer } \\
\hline miR-146b & 5'-ACACTCCAGCTGGGTGAGAACTGAATTCCA-3' & 5'-TGTCGTGGAGTCGGCAATTC-3' \\
U6 & 5'-CGCTTCGGCAGCACATATACTA-3' & 5'-ATGGAACGCTTCACGAATTTGC-3' \\
TNF- $\alpha$ & 5'-TGTTCCTCAGCCTCTTCTCCTT-3' & 5'-CTCTCAGCTCCACGCCATTG-3' \\
IL-6 & 5'-CTTCGGTCCAGTTGCCTTCTC-3' & 5'-AGGTGAGTGGCTGTCTGTGT-3' \\
IL-17 & 5'-ATTACTACAACCGATCCACCTCAC-3' & 5'-CCACGGACACCAGTATCTTCTC-3' \\
IL-22 & 5'-TATCACCAACCGCACCTTCAT-3' & 5'-CTCATACTGACTCCGTGGAACA-3' \\
GAPDH & 5'-GAGTCCACTGGCGTCTTCAC-3' & 5'-ATCTTGAGGCTGTTGTCATACTTCT-3'
\end{tabular}

TNF- $\alpha$, tumor necrosis factor $\alpha$; IL, interleukin; miR, microRNA.

Table II. Characteristics of the patients $(n=110)$.

\begin{tabular}{lc}
\hline Parameter & Value \\
\hline Age (years) & $47.4 \pm 10.8$ \\
Sex & \\
Male & $65(59.1)$ \\
Female & $45(40.9)$ \\
BMI (kg/m $\left.{ }^{2}\right)$ & $23.2 \pm 3.9$ \\
Disease duration (years) & $10.0(6.0-14.3)$ \\
Psoriasis-affected BSA $(\%)$ & $19.0 \pm 5.4$ \\
PASI score & $9.6 \pm 3.8$ \\
Clinical course, n (\%) & \\
Progressive stage & $59(53.6)$ \\
Quiescent stage & $24(21.8)$ \\
Regressive stage & $27(24.5)$ \\
Treatment & \\
Topical therapy & $99(90.0)$ \\
Phototherapy & $92(83.6)$ \\
Systemic non-biologic treatment & $77(70.0)$ \\
Systemic biologic treatment & $8(7.3)$ \\
\hline
\end{tabular}

Values are expressed as the mean \pm standard deviation, $\mathrm{n}(\%)$ or the median (interquartile range). BMI, body mass index; BSA, body surface area; PASI, psoriasis area and severity index.

was $19.0 \pm 5.4 \%$ and the PASI score was $9.6 \pm 3.8$ (Table II). The number of patients that received topical therapy, phototherapy, systemic non-biologic treatment and systemic biologic treatment was $99(90.0 \%), 92(83.6 \%), 77(70.0 \%)$ and $8(7.3 \%)$, respectively.

Comparison of miR-146b expression between psoriasis-affected tissue and unaffected tissue. The expression of miR-146b was increased in psoriasis-affected tissue when compared with that in unaffected tissue $(\mathrm{P}<0.001$; Fig. 1A). Furthermore, miR-146b was able to distinguish psoriasis-affected tissue from unaffected tissue with an AUC value of 0.781 (95\% CI: $0.720-0.843$; miR-146b cutoff value: 2.293 ; Fig. 1B) and the specificity and sensitivity was 96.4 and $53.6 \%$ respectively.
Correlation of miR-146b expression in psoriatic tissue with disease duration, psoriasis-affected BSA and PASI scores in patients with psoriasis. The results revealed that there was no correlation between miR-146b expression in psoriatic tissue and disease duration $(\mathrm{r}=0.058, \mathrm{P}=0.546$; Fig. $2 \mathrm{~A})$ or psoriasis-affected BSA ( $r=0.160, P=0.095$; Fig. 2B). However, miR-146b expression was positively correlated with the PASI score $(\mathrm{r}=0.423, \mathrm{P}<0.001$; Fig. $2 \mathrm{C})$, indicating that the expression of miR-146b was associated with increased disease activity in patients with psoriasis. In addition, the number of patients with psoriasis at the progressive, quiescent and regressive stage was 59, 24 and 27, respectively. The expression of miR-146b was highest in patients at the progressive stage, followed by that in patients at the quiescent stage and regressive stage $(\mathrm{P}<0.001$; Fig. S1).

Correlation of miR-146b expression in psoriatic tissue with treatment approaches in psoriasis patients. No correlation was identified between the expression of miR-146b in psoriatic tissue and topical therapy $(\mathrm{P}=0.608$; Fig. $3 \mathrm{~A})$, phototherapy $(\mathrm{P}=0.203$; Fig. 3B), systemic non-biologic treatment $(\mathrm{P}=0.589$; Fig. 3C) or systemic biologic treatment ( $\mathrm{P}=0.405$; Fig. 3D).

Correlation of miR-146b expression with inflammatory cytokine levels in psoriatic tissue. The expression of miR-146b in psoriatic tissue was determined to be positively correlated with the mRNA expression of TNF- $\alpha(r=0.403, \mathrm{P}<0.001$; Fig. 4A), IL-6 ( $\mathrm{r}=0.327, \mathrm{P}<0.001$; Fig. 4B) and IL-17 ( $\mathrm{r}=0.389$, $\mathrm{P}<0.001$; Fig. 4C). However, no significant correlation was obtained between miR-146b and IL-22 expression $(r=0.159$, $\mathrm{P}=0.098$; Fig. 4D). These results indicated that the expression of miR-146b was associated with elevated inflammation in psoriatic tissue.

Effect of miR-146b overexpression on cell proliferation and apoptosis of HaCaT cells. To investigate the potential role of miR-146b in the development of psoriasis, in vitro experiments were performed by transfecting miR-146b overexpression and control plasmids into $\mathrm{HaCaT}$ cells. The results revealed that the expression of miR-146b was significantly elevated following transfection of miR-146b overexpression plasmid $(\mathrm{P}<0.001$; Fig. 5A). This result indicated that plasmid-mediated overexpression was successful. Further experiments demonstrated that miR-146b overexpression enhanced cell proliferation 

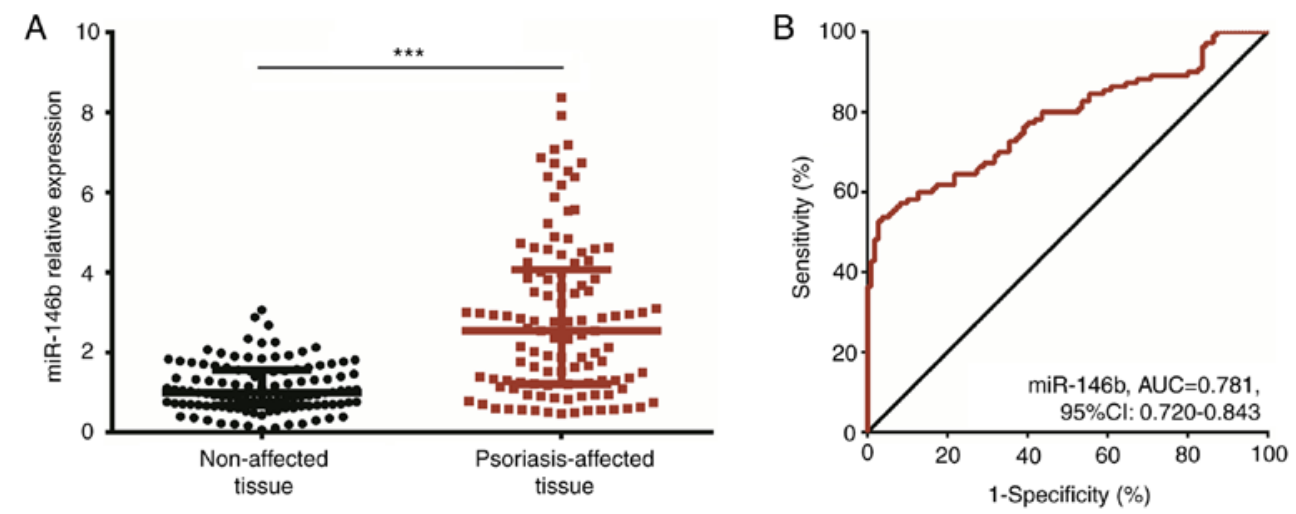

Figure 1. miR-146b expression in psoriasis-affected tissue and unaffected tissue. (A) miR-146b expression in psoriasis-affected tissue and unaffected tissue. (B) The ability of miR-146b expression to distinguish psoriasis-affected tissue from unaffected tissue was assessed using an ROC curve analysis. Comparisons between two individual groups were determined by Wilcoxon's rank-sum test. The ROC curve was drawn and the AUC was calculated to determine the ability of the parameter for distinguishing psoriasis-affected skin tissue from normal skin tissue. ${ }^{* * *} \mathrm{P}<0.001$. miR, microRNA; AUC, area under the curve; ROC, receiver operating characteristic.
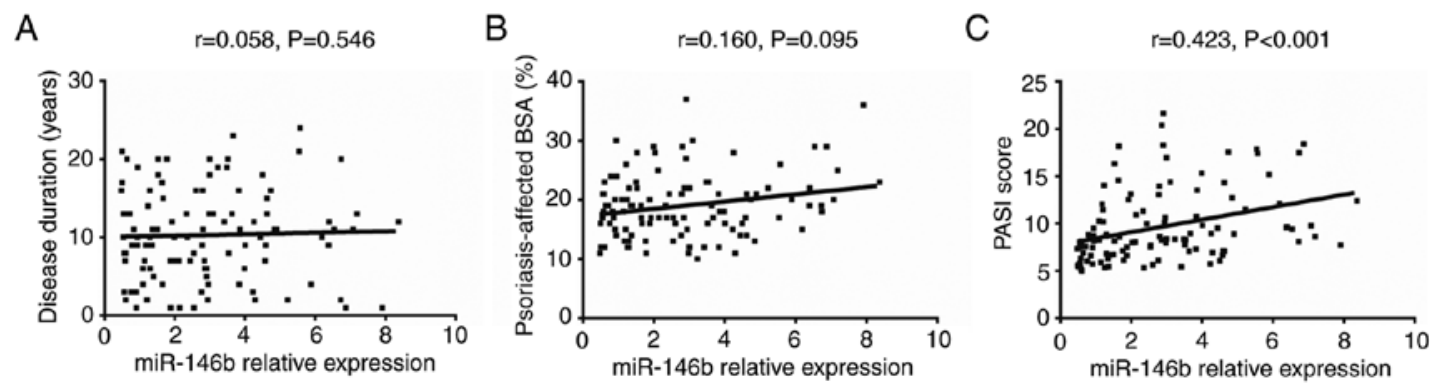

Figure 2. Association of miR-146b levels in psoriatic tissue with disease duration, psoriasis-affected BSA and PASI score in patients with psoriasis. Correlations between miR-146b expression in psoriatic tissue and (A) disease duration, (B) psoriasis-affected BSA and (C) PASI scores are presented. Correlations between two parameters were determined by Spearman correlation test. miR, microRNA; BSA, body surface area; PASI, psoriasis area and severity index.

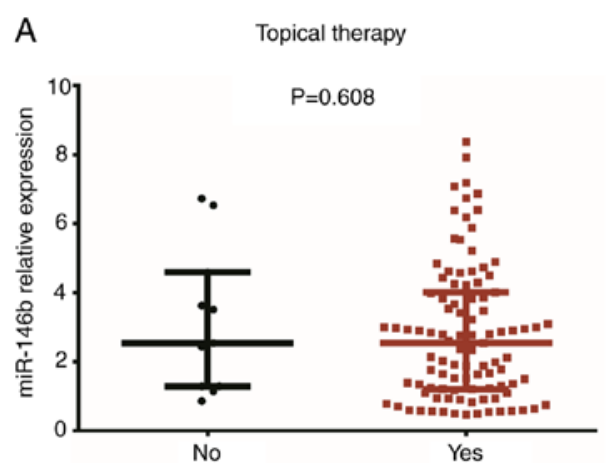

C

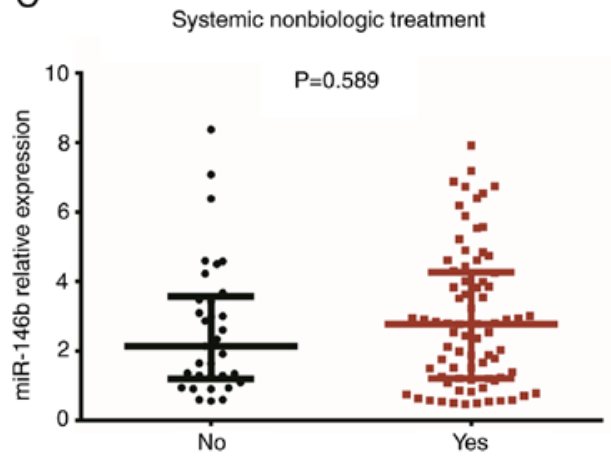

B Phototherapy

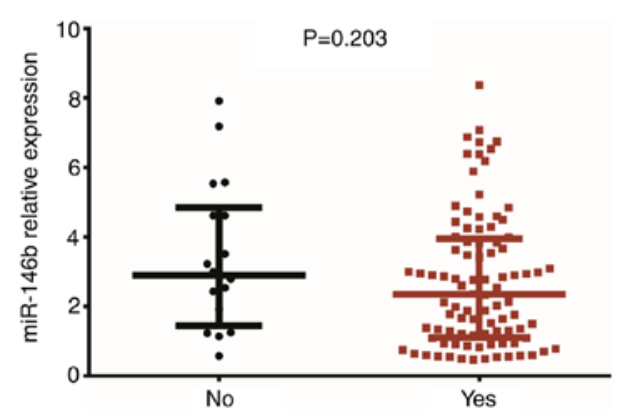

D

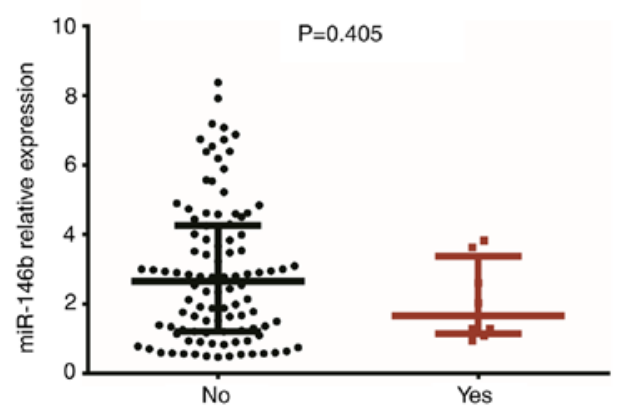

Figure 3. Association of miR-146b levels in psoriatic tissue with therapeutic approaches in patients with psoriasis. Correlations between miR-146b expression in psoriatic tissue and (A) topical therapy, (B) phototherapy, (C) systemic non-biological treatment or (D) systemic biologic treatment are presented. Comparisons between two individual groups were performed by Wilcoxon's rank-sum test. miR, microRNA. 

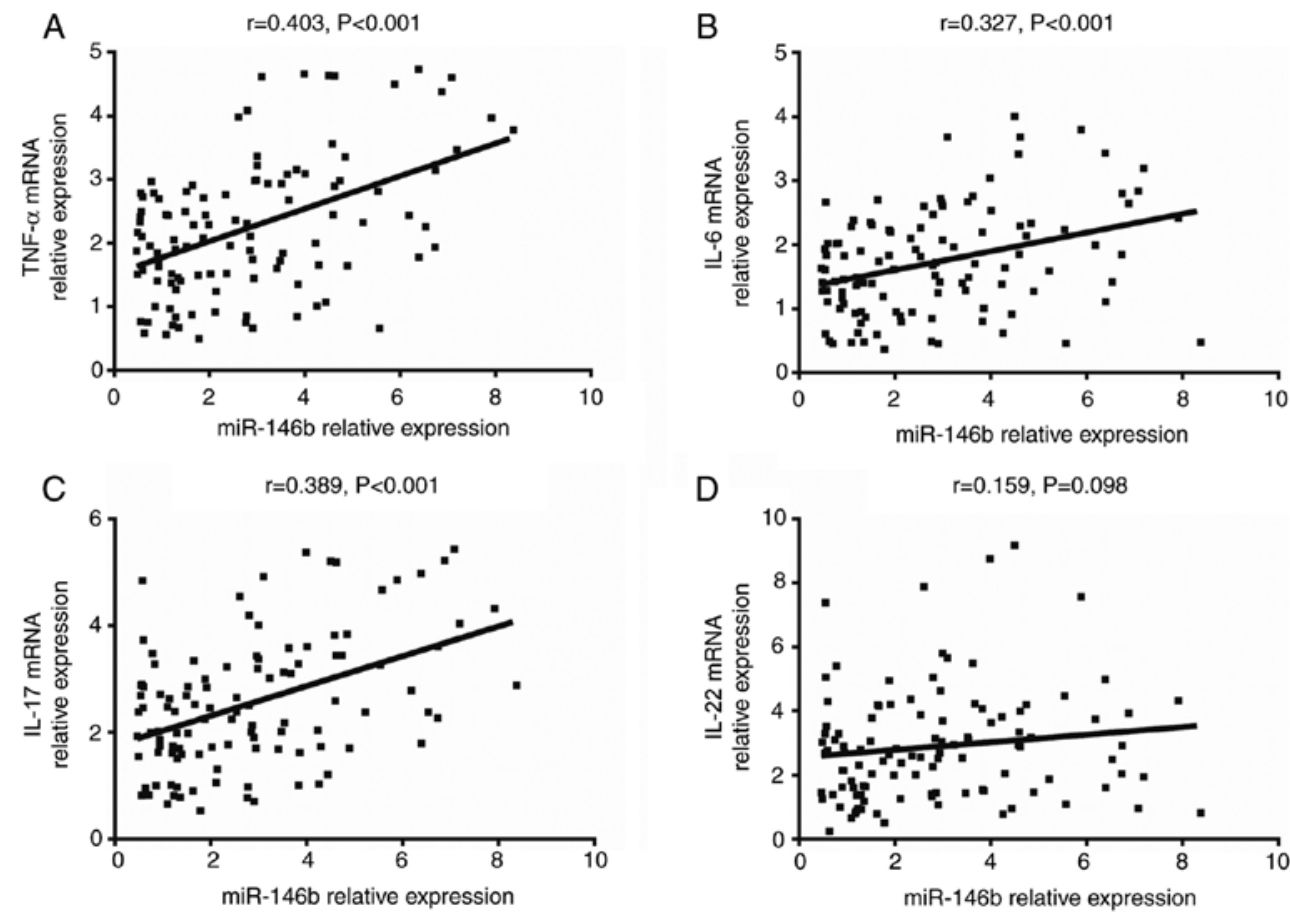

Figure 4. Association of miR-146b expression with inflammatory cytokine levels in psoriatic tissue. Correlations between miR-146b expression in psoriatic tissue and (A) TNF- $\alpha$, (B) IL-6, (C) IL-17 and (D) IL-22 mRNA expression are presented. Correlations between two parameters were determined by Spearman's correlation test. miR, microRNA; TNF, tumor necrosis factor; IL, interleukin.

at $48 \mathrm{~h}(\mathrm{P}<0.05)$ and $72 \mathrm{~h}(\mathrm{P}<0.01$; Fig. 5B), and suppressed cell apoptosis $(\mathrm{P}<0.01$; Fig. $5 \mathrm{C}$ and $\mathrm{D})$ compared with control $\mathrm{HaCaT}$ cells. To investigate the impact of miR-146b overexpression on keratinocytes under inflammatory conditions, HaCaT cells were treated with TRAIL following transfection. The results revealed that miR-146b overexpression attenuated TRAIL-induced cell apoptosis ( $\mathrm{P}<0.05$; Fig. 5E and F). Overall, it was indicated that miR-146b overexpression promoted cell proliferation and inhibited $\mathrm{HaCaT}$ cell apoptosis.

\section{Discussion}

In the present study, it was determined that the expression of miR-146b was increased in psoriasis-affected tissue when compared with that in unaffected tissue. The results also revealed that the expression of miR-146b was associated with elevated disease activity in patients with psoriasis and with aggravated inflammation in psoriatic tissue. Furthermore, overexpression of miR-146b enhanced keratinocyte proliferation, inhibited keratinocyte apoptosis and reduced TRAIL-induced keratinocyte apoptosis.

Numerous studies have demonstrated that miRNAs participate in various cell activities and signaling pathways. Investigating the role of these miRNAs in complex diseases (including psoriasis) may facilitate the discovery of novel biomarkers for their early diagnosis, also contributing to the elucidation of disease etiology. As a well-known miRNA, miR-146b is closely associated with inflammatory activities $(12,23,24)$. For instance, a previous study reported that miR-146b modulated the Toll-like receptor 4 signaling pathway by directly targeting myeloid differentiation primary response 88 (MYD88), TRAF6 and IL-1 receptor-associated kinase 1
(IRAK-1), thereby regulating pathogen detection and the initiation of the inflammatory response (25). A further study demonstrated that the upregulation of miR-146b attenuated bacterial lipopolysaccharide-induced inflammatory responses and reduced the expression of IRAK-1 and TRAF6 in human umbilical vein endothelial cells (26). In addition, miR-146b has been determined to be upregulated during human monocyte differentiation into immature dendritic cells (imDCs) and mature DCs (mDCs). Furthermore, silencing of miR-146b in imDCs and mDCs markedly prevented cell apoptosis, while miR-146b overexpression stimulated cell apoptosis (27). These studies indicated that miR-146b exerts multiple functions in the regulation of inflammation- and immune-associated signaling pathways.

Limited information exists regarding the clinical implications of miR-146b in autoimmune diseases. Only a small number of studies have reported that miR-146b is highly expressed in certain autoimmune conditions (including RA, giant cell arteritis and ATD), indicating that miR-146b may contribute to the pathology of autoimmune diseases $(17,18,28)$. Considering the potential role of miR-146b in the pathology of certain autoimmune diseases and the inflammation/autoimmune characteristics of psoriasis (a common and typical autoimmune disease), the present study hypothesized that miR-146b may also serve a role in the development and progression of psoriasis. To the best of our knowledge, only one study has determined that the expression of miR-146b was higher in psoriasis-affected skin compared with that in unaffected skin from patients with psoriasis and that of healthy controls. However, due to the small sample size of the aforementioned study $(n=30)$, the statistical power of this result was relatively low (29). Thus, further studies including 
A
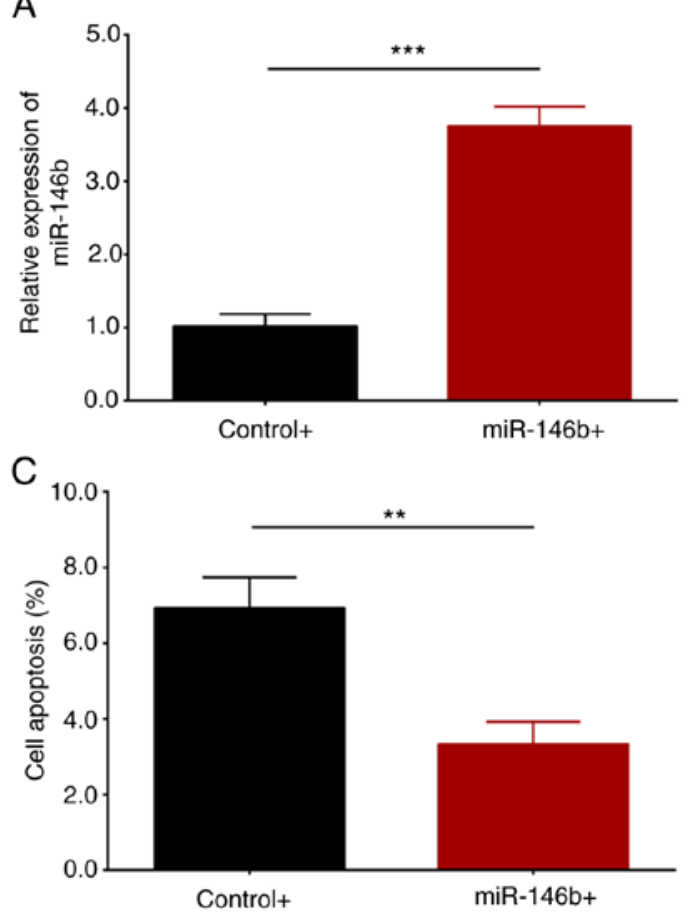

E

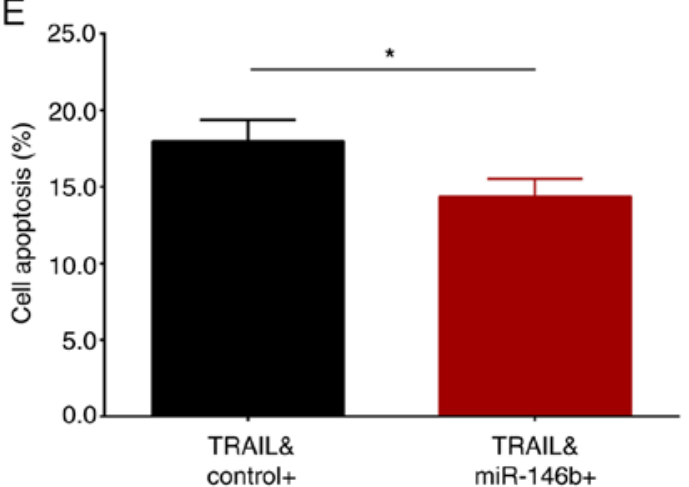

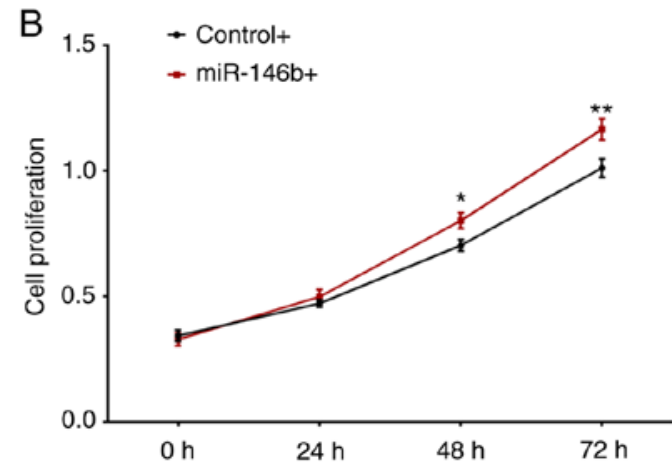

D

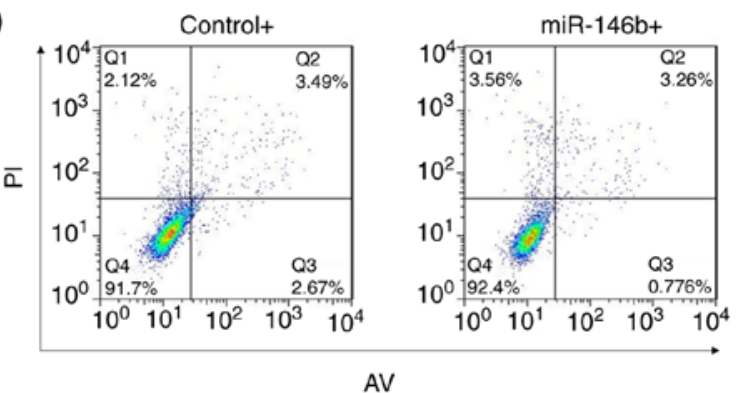

$\mathrm{F}$
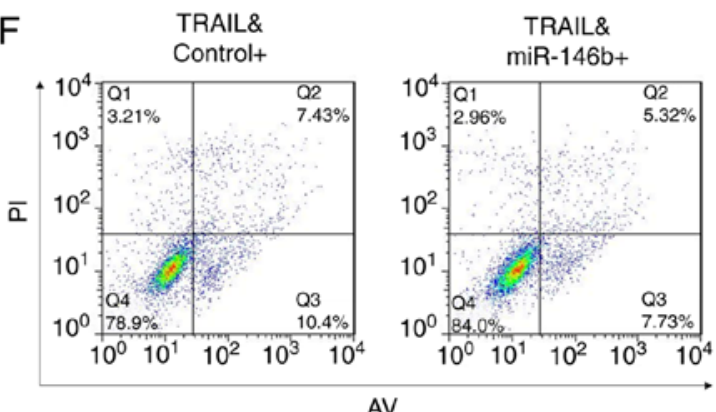

Figure 5. Impact of miR-146b overexpression on cell proliferation and apoptosis in HaCaT cells. (A) Comparison of miR-146b expression following miR-146b overexpression and control overexpression plasmid transfection in HaCaT cells. (B) Effect of miR-146b overexpression on HaCaT cell proliferation. (C and D) Apoptosis of HaCaT cells with miR-146b overexpression; (C) quantified results and (D) representative flow cytometry images. (E and F) TRAIL-induced cell apoptosis after miR-146b overexpression and control overexpression plasmid transfection; (E) quantified results and (F) representative flow cytometry images. Comparisons between two individual groups were determined using the t-test. ${ }^{*} \mathrm{P}<0.05,{ }^{* *} \mathrm{P}<0.01,{ }^{* * *} \mathrm{P}<0.001$. miR, microRNA; TRAIL, tumor necrosis factor-related apoptosis-inducing ligand; PI, propidium iodide; Q, quadrant; AV, annexin V.

more patients are essential. In the present study, the clinical implications of miR-146b in patients with psoriasis were investigated using 110 patients. The analyses revealed that miR-146b expression was increased in psoriasis-affected tissue when compared with that in unaffected tissue. Furthermore, miR-146b expression was highest in patients at the progressive stage, followed by patients at the quiescent stage and regressive stage. An explanation for these results may be that miR-146b regulates its target genes or proteins (including MYD88, TRAF6 and IRAK-1), which subsequently promote keratinocyte proliferation and inhibit keratinocyte apoptosis, thereby leading to the formation of psoriatic lesions and acceleration of disease progression (25). Thus, miR-146b expression was increased in psoriasis-affected tissue, and was also highly expressed in patients with psoriasis at the progressive stage.

According to previous studies, psoriasis is a recurrent, chronic autoimmune disease of the skin that involves the infiltration of white blood cells, abnormal proliferation of keratinocytes and activation of $\mathrm{T}$ cells in psoriasis-affected skin tissue, which secrete various cytokines (including TNF- $\alpha$, IL-6, IL-17 and IL-22) that commonly contribute to the pathological processes of psoriasis (2,30-33). Therefore, to explore the correlation between miRNA-146 expression and inflammation in patients with psoriasis, the expression of the most common inflammatory cytokines, including TNF- $\alpha$, IL-6, IL-17 and IL-22, were detected in the present study. The results revealed that miR-146b expression was correlated with higher PASI scores in patients with psoriasis and with elevated TNF- $\alpha$, IL- 6 and IL-17 mRNA expression in psoriatic tissue. These results indicated that miR-146b expression was correlated with increased disease activity and inflammation. The possible explanations for this result may be as follows: i) miR-146b mediates TRAF6 and IRAK-1 expression, promoting the release of pro-inflammatory 
cytokines (including TNF- $\alpha$, IL-6 and IL-17), thereby increasing disease activity and inflammation observed in patients with psoriasis $(12,34,35)$. ii) miR-146b may have activated several inflammation-associated signaling pathways (including NF- $\kappa \mathrm{B}$ ), causing a higher disease activity and aggravated inflammation in patients with psoriasis. Therefore, the present study indicated that miR-146b may serve as a novel biomarker for the predication of elevated psoriasis activity and aggravated inflammation in the clinical setting. However, the present study had certain limitations. First, psoriatic tissue was not easy to obtain, which means that it may not be feasible to predict disease activity levels based on measurements of miR-146b in the samples collected. Therefore, investigations into the predictive value of circulating miR-146b expression are required for determining psoriasis activity. In addition, the present study had a single-centered design, meaning that most of the patients were from North China, which may result in selection bias. Therefore, multi-center studies are required for future determinations.

Considering the malignant proliferation of keratinocytes in psoriatic tissue, and to further explore the underlying mechanism of miR-146b in the development of psoriasis, the miR-146b overexpression and control plasmids were transfected into HaCaT cells. The impact of miR-146b overexpression on $\mathrm{HaCaT}$ cell proliferation and apoptosis was subsequently evaluated. The present results revealed that miR-146b overexpression stimulated cell proliferation and suppressed cell apoptosis, indicating that miR-146b increased psoriasis activity and inflammation, potentially by enhancing keratinocyte proliferation and reducing keratinocyte apoptosis. To better understand the effect of miR-146b overexpression on keratinocytes under inflammatory conditions, HaCaT cell apoptosis following treatment with TRAIL (a protein that induces cell apoptosis) was also evaluated in the present study. The results demonstrated that miR-146b overexpression inhibited TRAIL-induced cell apoptosis. The reasons for this result may be as follows: i) miR-146b regulates multiple signaling pathways (including $\mathrm{NF}-\kappa \mathrm{B}$ ), thereby directly accelerating keratinocyte proliferation and inhibiting apoptosis $(23,36)$. ii) miR-146b mediates various proteins (including TRAF6 and IRAK-1) to promote the secretion of pro-inflammatory cytokines (including TNF- $\alpha$, IL-6 and IL-17), whose secretions may indirectly stimulate keratinocyte proliferation (37). Taken together, the present study provided novel insight into the pathogenesis of psoriasis and may facilitate the discovery of novel biomarkers for the prediction of psoriasis activity, as well as the discovery of novel treatment options.

In summary, miR-146b expression was elevated in psoriasis-affected tissue when compared with that in unaffected tissue, and its high expression was associated with increased disease activity in patients with psoriasis as well as aggravated inflammation in psoriatic tissue. Furthermore, miR-146b overexpression promoted keratinocyte proliferation and inhibited keratinocyte apoptosis in vitro.

\section{Acknowledgements}

Not applicable.

\section{Funding}

No funding was received.

\section{Availability of data and materials}

The datasets used and/or analyzed during the current study are available from the corresponding author on reasonable request.

\section{Authors' contributions}

XL conceived and designed the experiments. LZ and SZ performed the experiments. JW analyzed the data. SZ and JW wrote the main manuscript text. All authors revised the manuscript. All authors reviewed and approved the final manuscript.

\section{Ethics approval and consent to participate}

This study was approved by the Ethics Review Board of the Affiliated Hospital of Hebei University of Engineering (Handan, China) and all patients provided written informed consent.

\section{Patient consent for publication}

Not applicable.

\section{Competing interests}

The authors declare that they have no competing interests.

\section{References}

1. Parisi R, Symmons DP, Griffiths CE and Ashcroft DM; Identification and Management of Psoriasis and Associated ComorbidiTy (IMPACT) project team: Global epidemiology of psoriasis: A systematic review of incidence and prevalence. J Invest Dermatol 133: 377-385, 2013.

2. von Csiky-Sessoms S and Lebwohl M: What's new in psoriasis. Dermatol Clin 37: 129-136, 2019.

3. Christophers E and van de Kerkhof PCM: Severity, heterogeneity and systemic inflammation in psoriasis. J Eur Acad Dermatol Venereol 33: 643-647, 2019.

4. Golińska J, Sar-Pomian M and Rudnicka L: Dermoscopic features of psoriasis of the skin, scalp and nails-a systematic review. J Eur Acad Dermatol Venereol 33: 648-660, 2019.

5. Hwang YJ, Jung HJ, Kim MJ, Roh NK, Jung JW, Lee YW, Choe YB and Ahn KJ: Serum levels of LL-37 and inflammatory cytokines in plaque and guttate psoriasis. Mediators Inflamm 2014: 268257, 2014.

6. Menter A, Strober BE, Kaplan DH, Kivelevitch D, Prater EF, Stoff B, Armstrong AW, Connor C, Cordoro KM, Davis DMR, et al: Joint AAD-NPF guidelines of care for the management and treatment of psoriasis with biologics. J Am Acad Dermatol 80: 1029-1072, 2019.

7. Elmets CA, Leonardi CL, Davis DMR, Gelfand JM, Lichten J, Mehta NN, Armstrong AW, Connor C, Cordoro KM, Elewski BE, et al: Joint AAD-NPF guidelines of care for the management and treatment of psoriasis with awareness and attention to comorbidities. J Am Acad Dermatol 80: 1073-1113, 2019.

8. Guo WT and Wang Y: Dgcr8 knockout approaches to understand microRNA functions in vitro and in vivo. Cell Mol Life Sci 76: 1697-1711, 2019.

9. Saliminejad K, Khorram Khorshid HR, Soleymani Fard S and Ghaffari SH: An overview of microRNAs: Biology, functions, therapeutics, and analysis methods. J Cell Physiol 234: 5451-5465, 2019. 
10. Asadzadeh Z, Mansoori B, Mohammadi A, Aghajani M, Haji-Asgarzadeh K, Safarzadeh E, Mokhtarzadeh A, Duijf PHG and Baradaran B: microRNAs in cancer stem cells: Biology, pathways, and therapeutic opportunities. J Cell Physiol 234: 10002-10017, 2019.

11. Basso MF, Ferreira PCG, Kobayashi AK, Harmon FG, Nepomuceno AL, Molinari HBC and Grossi-de-Sa MF: MicroRNAs and new biotechnological tools for its modulation and improving stress tolerance in plants. Plant Biotechnol J 17: $1482-1500,2019$

12. Hou T, Liao J, Zhang C, Sun C, Li X and Wang G: Elevated expression of miR-146, miR-139 and miR-340 involved in regulating Th1/Th2 balance with acute exposure of fine particulate matter in mice. Int Immunopharmacol 54: 68-77, 2018.

13. Tu Z, Xiong J, Xiao R, Shao L, Yang X, Zhou L, Yuan W, Wang M, Yin Q, Wu Y, et al: Loss of miR-146b-5p promotes $T$ cell acute lymphoblastic leukemia migration and invasion via the IL-17A pathway. J Cell Biochem 120: 5936-5948, 2019.

14. Kirchmeyer M,Servais FA, Hamdorf M, Nazarov PV, Ginolhac A, Halder R, Vallar L, Glanemann M, Rubie C, Lammert F, et al: Cytokine-mediated modulation of the hepatic miRNome: miR-146b-5p is an IL-6-inducible miRNA with multiple targets. J Leukoc Biol 104: 987-1002, 2018.

15. Yan M, Yang X, Shen R, Wu C, Wang H, Ye Q, Yang P, Zhang L, Chen M, Wan B, et al: miR-146b promotes cell proliferation and increases chemosensitivity, but attenuates cell migration and invasion via FBXL10 in ovarian cancer. Cell Death Dis 9: 1123, 2018.

16. Yu C, Zhang L, Luo D, Yan F, Liu J, Shao S, Zhao L, Jin T, Zhao J and Gao L: MicroRNA-146b-3p promotes cell metastasis by directly targeting NF2 in human papillary thyroid cancer. Thyroid 28: 1627-1641, 2018.

17. Nakasa T, Miyaki S, Okubo A, Hashimoto M, Nishida K, Ochi M and Asahara $\mathrm{H}$ : Expression of microRNA-146 in rheumatoid arthritis synovial tissue. Arthritis Rheum 58: 1284-1292, 2008.

18. Martínez-Hernández R,Sampedro-Núñez M,Serrano-Somavilla A, Ramos-Leví AM, de la Fuente H, Triviño JC, Sanz-García A, Sánchez-Madrid F and Marazuela M: A MicroRNA signature for evaluation of risk and severity of autoimmune thyroid diseases. J Clin Endocrinol Metab 103: 1139-1150, 2018.

19. Venkatesha SH, Dudics S, Song Y, Mahurkar A and Moudgil KD: The miRNA expression profile of experimental autoimmune encephalomyelitis reveals novel potential disease biomarkers. Int J Mol Sci 19: 3990, 2018

20. Wallace AB: The exposure treatment of burns. Lancet 1: 501-504, 1951.

21. Fredriksson T, Pettersson U: Severe psoriasis-oral therapy with a new retinoid. Dermatologica 157: 238-244, 1978.

22. Livak KJ and Schmittgen TD: Analysis of relative gene expression data using real-time quantitative PCR and the 2(-Delta Delta C(T)) method. Methods 25: 402-408, 2001

23. Cho S, Lee HM, Yu IS, Choi YS, Huang HY, Hashemifar SS Lin LL, Chen MC, Afanasiev ND, Khan AA, et al: Differential cell-intrinsic regulations of germinal center $B$ and $T$ cells by miR-146a and miR-146b. Nat Commun 9: 2757, 2018

24. Zhang Z, Dai X, Qi J, Ao Y, Yang C and Li Y: Astragalus mongholicus (Fisch.) bge improves peripheral treg cell immunity imbalance in the children with viral myocarditis by reducing the levels of miR-146b and miR-155. Front Pediatr 6: $139,2018$.
25. Curtale G, Mirolo M, Renzi TA, Rossato M, Bazzoni F and Locati M: Negative regulation of Toll-like receptor 4 signaling by IL-10-dependent microRNA-146b. Proc Natl Acad Sci USA 110: 11499-11504, 2013

26. Echavarria R, Mayaki D, Neel JC, Harel S, Sanchez V and Hussain SN: Angiopoietin-1 inhibits toll-like receptor 4 signalling in cultured endothelial cells: Role of miR-146b-5p. Cardiovasc Res 106: 465-477, 2015.

27. Park H, Huang X, Lu C, Cairo MS and Zhou X: MicroRNA-146a and microRNA-146b regulate human dendritic cell apoptosis and cytokine production by targeting TRAF6 and IRAK1 proteins. J Biol Chem 290: 2831-2841, 2015.

28. Croci S, Zerbini A, Boiardi L, Muratore F, Bisagni A, Nicoli D, Farnetti E, Pazzola G, Cimino L, Moramarco A, et al: MicroRNA markers of inflammation and remodelling in temporal arteries from patients with giant cell arteritis. Ann Rheum Dis 75: 1527-1533, 2016.

29. Hermann H, Runnel T, Aab A, Baurecht H, Rodriguez E, Magilnick N, Urgard E, Sahmatova L, Prans E, Maslovskaja J, et al: miR-146b probably assists miRNA-146a in the suppression of keratinocyte proliferation and inflammatory responses in psoriasis. J Invest Dermatol 137: 1945-1954, 2017.

30. Grine L, Dejager L, Libert C and Vandenbroucke RE: An inflammatory triangle in psoriasis: TNF, type I IFNs and IL-17. Cytokine Growth Factor Rev 26: 25-33, 2015.

31. Wang WM and Jin HZ: Interleukin-6 in psoriasis. Zhongguo Yi Xue Ke Xue Yuan Xue Bao 40: 284-288, 2018 (In Chinese).

32. Mease PJ: Inhibition of interleukin-17, interleukin-23 and the TH17 cell pathway in the treatment of psoriatic arthritis and psoriasis. Curr Opin Rheumatol 27: 127-133, 2015.

33. Hao JQ: Targeting interleukin-22 in psoriasis. Inflammation 37: 94-99, 2014.

34. Viladomiu M, Hontecillas R, Pedragosa M, Carbo A, Hoops S, Michalak P, Michalak K, Guerrant RL, Roche JK, Warren CA and Bassaganya-Riera $\mathrm{J}$ : Modeling the role of peroxisome proliferator-activated receptor $\gamma$ and microRNA-146 in mucosal immune responses to Clostridium difficile. PLoS One 7: e47525, 2012.

35. Cai F, Wu F, Cao J and Chen X: MicroRNA-146b-3p regulates the development and progression of cerebral infarction with diabetes through RAF1/P38MAPK/COX-2 signaling pathway. Am J Transl Res 10: 618-628, 2018.

36. Weng Z, Patel AB, Vasiadi M, Therianou A and Theoharides TC: Luteolin inhibits human keratinocyte activation and decreases $\mathrm{NF}-\kappa \mathrm{B}$ induction that is increased in psoriatic skin. PLoS One 9: e90739, 2014

37. Nestle FO, Kaplan DH and Barker J: Psoriasis. N Engl J Med 361: 496-509, 2009.

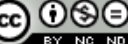

This work is licensed under a Creative Commons Attribution-NonCommercial-NoDerivatives 4.0 International (CC BY-NC-ND 4.0) License. 